\title{
Generalized Oppositional Moth Flame Optimization with Crossover Strategy: An Approach for Medical Diagnosis
}

\author{
Jianfu Xia ${ }^{1}$, Hongliang Zhang ${ }^{2}$, Rizeng Li ${ }^{1 *}$, Huiling Chen ${ }^{2 *}$, Hamza Turabieh ${ }^{3}$, Majdi Mafarja ${ }^{4}$, Zhifang Pan ${ }^{5^{*}}$ \\ 1. Department of General Surgery, The Second Affiliated Hospital of Shanghai University, Wenzhou 325000, China \\ 2. Department of Computer Science and Artificial Intelligence, Wenzhou University, Wenzhou 325035, China \\ 3. Department of Information Technology, College of Computers and Information Technology, P.O. Box11099, \\ Taif 21944, Taif University, Taif, Saudi Arabia \\ 4. Department of Computer Science, Birzeit University, Ramallah 72439, Palestine \\ 5. The First Affiliated Hospital of Wenzhou Medical University, Wenzhou 325000, China
}

\begin{abstract}
In the original Moth-Flame Optimization (MFO), the search behavior of the moth depends on the corresponding flame and the interaction between the moth and its corresponding flame, so it will get stuck in the local optimum easily when facing the multi-dimensional and high-dimensional optimization problems. Therefore, in this work, a generalized oppositional MFO with crossover strategy, named GCMFO, is presented to overcome the mentioned defects. In the proposed GCMFO, GOBL is employed to increase the population diversity and expand the search range in the initialization and iteration jump phase based on the jump rate; crisscross search (CC) is adopted to promote the exploitation and/or exploration ability of MFO. The proposed algorithm's performance is estimated by organizing a series of experiments; firstly, the CEC2017 benchmark set is adopted to evaluate the performance of GCMFO in tackling high-dimensional and multimodal problems. Secondly, GCMFO is applied to handle multilevel thresholding image segmentation problems. At last, GCMFO is integrated into kernel extreme learning machine classifier to deal with three medical diagnosis cases, including the appendicitis diagnosis, overweight statuses diagnosis, and thyroid cancer diagnosis. Experimental results and discussions show that the proposed approach outperforms the original MFO and other state-of-the-art algorithms on both convergence speed and accuracy. It also indicates that the presented GCMFO has a promising potential for application.
\end{abstract}

Keywords: nature-inspired algorithm, moth-flame optimization, generalized opposition-based learning, crisscross search, medical diagnosis

Copyright (C) The author(s) 2021.

\section{Introduction}

More and more Swarm Intelligence (SI) based algorithms have arisen in recent years ${ }^{[1,2]}$. SI-based algorithms can be a solution to many problems like medical $\operatorname{diagnosis}^{[3-6]}$, financial distress prediction ${ }^{[7-9]}$, energy field ${ }^{[10-12]}$, engineering problems ${ }^{[13-19]}$, feature reduction $^{[20,21]}$, educational field ${ }^{[22-24]}$, maximum satisfiability problem $^{[25,26]}$, PID optimization control ${ }^{[27-29]}$, wind speed prediction ${ }^{[30]}$, fault diagnosis of rolling bearings ${ }^{[31,32]}$, gate resource allocation ${ }^{[33,34]}$ and scheduling problem $^{[35,36]}$. SI based algorithms can be classified into two categories: the environment inspires one kind, such as Particle Swarm Optimization (PSO) ${ }^{[37]}$, Artificial Bee Colony $(\mathrm{ABC})^{[38]}$, and so on; another kind is inspired by social behavior, for example, Moth-Flame Optimization
$(\mathrm{MFO})^{[39]}$, Harris Hawks Optimizer $(\mathrm{HHO})^{[40]}$, Slime Mould Algorithm (SMA ${ }^{[41]}$, etc. As a classical SI-based algorithm, inspired by the behavior of moths called transverse orientation, MFO was established for real engineering optimization problems by Mirjalilili ${ }^{[39]}$.

Since it was established, many scholars have employed various strategies to enhance the convergence speed and accuracy of MFO to obtain stronger MFO varieties. One way is to integrate local search and/or global search mechanisms to boost exploration and/or exploitation of original MFO. A new enhanced MFO named OMFO was presented by introducing OBL, Cauchy Mutation (CM), and Evolution Boundary Constraint Handling $(\mathrm{EBCH})^{[42]}$. Chaotic maps had been utilized to replace the parameters of MFO based on their randomicity to enhance the exploration and/or exploita-

\footnotetext{
*Corresponding author: Rizeng Li, Huiling Chen, Zhifang Pan

E-mail: 13857761117@163.com, chenhuiling.jlu@gmail.com, panzhifang@wmu.edu.cn
} 
tion capability of the original $\mathrm{MFO}^{[3,43-45]}$. Lévy-Flight was also adopted to boost the exploration and/or exploitation ability of $\mathrm{MFO}^{[46-48]}$. Zhang et al. integrated orthogonal learning and Broyden-FletcherGoldfarb-Shanno into MFO to enhance its exploration and exploitation and applied it to constrained engineering optimization problems ${ }^{[49]}$.

Another way is to combine the characteristic of other EAs with the feature of the original MFO to heighten the performance of basic MFO. The specific search mechanism of advanced EAs can make up for the shortcomings of basic MFO. For example, PSO was integrated into MFO to accelerate its convergence speed and improve its accuracy ${ }^{[50]}$. A new kind of nature-inspired algorithm Water Cycle Algorithm (WCA) was also introduced into MFO to enhance the exploitation capability of $\mathrm{MFO}^{[51,52]}$. Firefly Algorithm (FA) was integrated into MFO to enhance the exploitation ability of $\mathrm{MFO}^{[53]}$. Gaussian mutation and opposition-based learning were introduced into MFO to boost its performance ${ }^{[54]}$. Chen et al. introduced the Sine Cosine Algorithm (SCA) feature into MFO to enhance its exploration performance ${ }^{[55]}$.

Many scholars have improved MFO to make it suitable for suit binary problems and multi-objective problems. Such as Binary-coded Modified Moth Flame Optimization Algorithms (BMMFOA) to solve unit commitment problems existing in power system operational scheduling ${ }^{[56]}$. The mixed-integer optimization problems were solved by $\mathrm{MFO}^{[57]}$. A Non-dominated Sorting Moth-Flame Optimization algorithm (NS-MFO) was presented for multi-objective problems based on the elitist non-dominated sorting and crowding distance approach $^{[58]}$. MFO and other bio-inspired algorithms were utilized to deal with wind integrated multi-objective optimal power dispatch (MOOD) problems ${ }^{[59]}$.

Since it was proposed, MFO has been applied to solve various engineering problems. Zhang et al. introduced orthogonal learning and Nelder-Mead simplex with MFO to tackle photovoltaic modules' parameters and achieve competitive results ${ }^{[11]}$. MFO was combined with Neutrosophic Sets (NS) for breast cancer medical diagnosis ${ }^{[60]}$. MFO was used to evaluate the schedule plans of the regionally integrated energy system ${ }^{[61]}$.
MFO was also adopted to evaluate the parameters of machine learning algorithms ${ }^{[3,62-64]}$. Li et al. combined feature selection with MFO based SVM to predict the diagnosis of tuberculous pleural effusion ${ }^{[65]}$. Xu et al. combined improved binary moth-flame optimization algorithm with an extreme learning machine to predict phenanthrene toxicity on mice ${ }^{[66]}$.

Deng et al. integrated three improved strategies: differential mutation, multi-population mutation, and feasible solution space transformation to boost the optimization performance of MSIQDE ${ }^{[67]}$. Niche co-evolution mechanism cooperated with enhanced particle swarm optimization algorithm to enhance the performance of the Quantum Evolutionary Algorithm (QEA) in solving multi-objective optimization problems ${ }^{[34]}$. The OBL, multi-population parallel control, and co-evolutionary mutation were employed to strengthen the optimization capability in identifying the parameters of photovoltaic (PV) models ${ }^{[68]}$. The optimal mutation mechanism, the wavelet basis, and normal distribution functions were embedded into differential evolution to accurate its convergence speed and accuracy in dealing with function optimization and airport gate assignment problem ${ }^{[69]}$. The greedy mutation mechanism was adopted to enhance the performance of the enhanced success history adaptive DE (LSHADE) in optimizing the parameter of PV models ${ }^{[70]}$.

Generalized opposition-based learning has been proved that can produce a higher quality population in the Initialization and iteration jump phase by many scholars, meanwhile, crisscross search can enhance the global convergence of SI-based algorithms such as $\mathrm{PSO}^{[71,72]}$ and $\mathrm{DE}^{[73]}$. Therefore, this paper presented an enhanced moth-flame optimization called GCMFO by embedding crisscross search and generalized oppositional-based learning to accelerate the optimization capability of MFO. At the same time, the proposed GCMFO is applied in function optimization, and the real-life medical diagnosis cases. The contribution of this work can be summarized as follows:

(1) Crisscross search technique can boost the exploration capacity of each agent in the population. Hence the optimization performance of MFO has been accelerated.

(2) Generalized opposition-based learning can in- 
crease the diversity of the population in MFO; at the same time, it expands the search area in the search process so that it can avoid premature convergence effectively.

(3) The designed framework makes the balance between exploitation and exploration achieve a steadier condition.

(4) GCMFO can be treated as a promising solving method for medical diagnosis.

The main work of this paper is described as follows: the fundamental of MFO, crisscross search and generalized opposition-based learning are demonstrated in section 2. The implementation details and framework of the proposed GCMFO are shown in section 3. The experiment results and discussion about CEC2017 benchmark problems, and medical diagnosis are described in detail to confirm the performance of our method in section 4. At last, the conclusions and future works are summarized in section $\mathbf{5}$.

\section{Background}

\subsection{Moth-Flame Optimization (MFO)}

An interesting phenomenon is that moths navigate to the moon, lights, or other luminous objects on the spiral trajectory to keep the fixed orientation with the target object. Inspired by this mechanism, Mirjalili established MFO to solve global optimization problems ${ }^{[39]}$. There are two kinds of individuals, and one is called moths; the other is flames. In MFO, moths denote the candidate solutions in the search space; at the same time, flames indicate the obtained optimal solutions. The main operation steps of MFO are described as follows.

Firstly, generating the initial swarm of moths randomly, calculating the fitness of moth population, and tagging the sorted initial moth swarm in the ascending order according to fitness as an initial flame population.

Then, decreasing the flame population size to the determined number $R$ linearly, updating the location of each moth depending its corresponding flame based on the spiral curve function. Merging the updated moth population into the current flame population, selecting the $R$ best agents as the new flame population for the next iteration. The process of updating the moth population and the flame population will be repeated until the termination condition is met.

At last, when the termination condition is reached, outputting the best flame that denotes the global best solution. The pseudocode of the MFO is shown in the supplementary material.

\subsection{Generalized opposition-based learning (GOBL)}

In 2005, Tizhoosh proposed the concept of opposition-based Learning, named OBL for the computational intelligence field ${ }^{[74]}$; since Tizhoosh, OBL has been proved to enhance population diversity the exploration ability of $\mathrm{S}^{[74]}$. Wang et al. presented the variant version of OBL generalized opposition-based learning ${ }^{[75]}$, called GOBL, and applied it to accelerate the performance of $\mathrm{DE}^{[76,77]}, \mathrm{PSO}^{[75]}, \mathrm{ABC}^{[78,79]}$, and so on.

\subsubsection{GOBL-based population initialization}

In the population initialization stage, GOBL is utilized to calculate the opposite position of the agents in the initial population $X$ with $N$ agents, the opposite positions of the individuals in the initial population construct the opposite population of the initial population $X^{G O}$ based on the Eq. (1), and Eq. (2). The $N$ best individuals are selected from the mixed population formed by the initial population $M$ and the opposite population $M^{G O}$ as the new initial population to participate in the next optimization process. In this phase, GOBL can make the algorithm obtain a better initial population that can lead the population to find a better optimal solution with high probability.

$$
\begin{gathered}
M_{i, j}^{G O}=k *\left(l b_{j}+u b_{j}\right)-M_{i, j}, \\
M_{i, j}^{G O}=\operatorname{rand}\left(l b_{j}, u b_{j}\right), \text { if } M_{i, j}^{G O} \notin\left(l b_{j}, u b_{j}\right),
\end{gathered}
$$

where $i=1,2, \ldots, N ; j=1,2, \ldots, D ; M_{i, j}$ denotes the $j$ th dimension of the $i$ th individual in the population; $M_{i, j}^{G O}$ indicates the opposite location of $M_{i, j} ; l b_{j}$ and $u b_{j}$ are the $j$ th dimensions of the lower boundary and upper boundary of the feasible region in the given problem; $k$ is a random number in the range $[0,1] ; N$ means the population size; $D$ is the dimension value of the given problem. If the opposite position of the individual is out of the search space, they will be randomly regenerated within the search space $(l b, u b)$. 


\subsubsection{GOBL-based population optimization}

During the iterative optimization process, GOBL is used to generate the opposite population $M^{G O}$ of the current population $M$ by calculating the opposite positions of the agents in the current population-based on Eqs. (3) - (5). Merge the current population $M$ and the opposite population $M^{G O}$ into a hybrid population $M \cup M^{G O}$, then select $N$ best agents from the hybrid population $M \cup M^{G O}$ to update the current population and get into the next iteration. In this stage, GOBL can avoid the population trapping in the local optimum and expand the search region for the population.

$$
\begin{gathered}
a_{j}=\min \left(X_{i, j}\right), b_{j}=\max \left(X_{i, j}\right), \\
M_{i, j}^{G O}=k *\left(a_{j}+b_{j}\right)-M_{i, j}, \\
M_{i, j}^{G O}=\operatorname{rand}\left(a_{j}, b_{j}\right), \text { if } M_{i, j}^{G O} \notin\left(a_{j}, b_{j}\right),
\end{gathered}
$$

where $a_{j}$ and $b_{j}$ are the $j$ th dimensions of the dynamic lower boundary and upper boundary of the current population, they are determined by the current population distribution; $M_{i, j}$ means the $j$ th dimension of the agent in the current population; $k$ is a random number in the range $[0,1] ; N$ means the population size; $D$ is the dimension value of the given problem. If the opposite position of the individual is out of the search space, they will be regenerated within the search space $(a, b)$ randomly.

\subsection{Crisscross search optimization (CC)}

Inspired by the golden mean concept in Confucianism and the crossover operator in GA, Meng et al. presented a crisscross optimization algorithm $(\mathrm{CSO})^{[80]}$ and applied it in large-scale economic dispatch problems in the electric power system ${ }^{[81-83]}$. In CSO, the key mechanism is crisscross search (CC), containing vertical crossover and horizontal crossover. Zhao et al. introduced the $\mathrm{CC}$ mechanism into ant colony optimization to deal with multi-threshold image segmentation ${ }^{[84]}$. Liu et al. combined the CC strategy with $\mathrm{HHO}$ to estimate the parameters of photovoltaic models ${ }^{[85]}$. They all acquired promising results and verified the effect of $\mathrm{CC}$ in the search process.

\subsubsection{Vertical crossover search}

The vertical crossover can enhance the population diversity and avoid some dimensions trapping into stagnation. It can be realized by carrying out the arithmetic crossover on all agents between two diverse dimensions. If the $j 1$ th and $j 2$ th dimensions of the $i$ th agent are selected to perform the vertical crossover, so a new offspring $M v c_{i}$ can be generated based on Eq. (6) and Eq. (7).

$$
\begin{gathered}
M v c_{i, j 1}=r * M_{i, j 1}+(1-r) * M_{i, j 2}, \\
r=\text { unifrnd }(0,1),
\end{gathered}
$$

where $i=1,2, \ldots, N ; j 1, j 2=1,2, \ldots, D ; M v c_{i, j 1}$ denotes the $j 1$ th dimension of the offspring $M v c_{i} . M_{i, j 1}$, and $M_{i, j 2}$ indicate $j 1$ th and $j 2$ th dimensions of the $i$ th agent $M_{i} . r$ is a uniformly distributed random number in the region $[0,1]$.

Note that it should carry out normalization operation based on each dimension's lower and upper boundary before the vertical crossover operation to ensure the offspring generated by the vertical crossover operation distribute in the boundary of each dimension. After performing the vertical crossover, carry out the reverse normalization operation to guarantee the offspring locate in the boundary of the given problem. The pseudocode of the vertical crossover is illustrated in the supplementary material.

\subsubsection{Horizontal crossover search}

The horizontal crossover can accelerate the exploration and exploitation of the search agent. It is implemented by performing an arithmetic crossover operation on all dimensions between two diverse agents. If a pair of agents $M_{i 1}$ and $M_{i 2}$ are chosen to perform the horizontal crossover, the two offspring $M h c_{i 1}$ and $M h c_{i 2}$ of $M_{i 1}$ and $M_{i 2}$ can be produced by Eq. (8) and Eq. (9), respectively.

$$
M h c_{i 1, j}=r 1 * M_{i 1, j}+(1-r 1) * M_{i 2, j}+c 1 *\left(M_{i 1, j}-M_{i 2, j}\right) \text {, }
$$

$$
M h c_{i 2, j}=r 1 * M_{i 2, j}+(1-r 1) * M_{i 1, j}+c 2 *\left(M_{i 2, j}-M_{i 1, j}\right) \text {, }
$$

where $M h c_{i 1, j}$, and $M h c_{i 2, j}$ are the $j$ th dimension of $M h c_{i 1}$ and $M h c_{i 2} . M_{i 1, j}$, and $M_{i 2, j}$ denote the $j$ th dimension of $M_{i 1}$ and $M_{i 2} . r 1$ and $r 2$ are uniformly distributed random numbers in the region $(0,1] . c 1$ and $c 2$ are uniformly 
distributed random numbers in the region $[-1,1]$. The pseudocode of the horizontal crossover is demonstrated in the supplementary material.

\section{The proposed GCMFO}

Based on the above background knowledge, the implementation and analysis of computational time complexity are described below.

\subsection{The implementation of GCMFO}

In this work, GOBL and $\mathrm{CC}$ are both embedded into the original MFO to boost its optimization performance, GOBL strategy is utilized in the initialization phase to improve the quality of the initial population, and on the iteration jump stage to increase the population diversity and expand the search area, the cooperation between GOBL and CC makes the exploration and exploitation of the proposed GCMFO reach a steadier status. The implementation details are described step by step as below.

Step 1: Initialize the relevant parameter in the presented GCMFO, such as population size $N$, the problem dimension $D$, the lower boundary $l b$ and the upper boundary $u b$ of the solution space, the maximum fitness evaluations MaxFEs, the control coefficient $p 2$ in vertical crossover search, the jump rate $J r$ for GOBL during the iteration jump.

Step 2: Initialize the location of each moth $M_{i}$ in the population randomly within the lower boundary $l b$ and the upper boundary $u b$ by Eq. (10), the current fitness evaluations $F E s=0$, the current iteration $t=1$.

$$
M_{i, j}=l b_{j}+\operatorname{rand}() *\left(u b_{j}-l b_{j}\right)
$$

where $i=1,2, \ldots, N, j=1,2, \ldots, D$.

Step 3: Evaluate the moth population, $F E s=F E s+N$; perform GOBL-based population initialization strategy to obtain new initial moth population, $F E s=F E s+N$.

Step 4: Calculate the flame number $R$ and the parameter $a$ according to Eqs. (11) and (12), respectively.

$$
\begin{gathered}
R=\operatorname{round}(N-F E s *(N-1) / \text { MaxFEs }), \\
\quad a=-1+F E s *(-1 / \text { MaxFEs }),
\end{gathered}
$$

Step 5: If $t==1$, sort the initial moth population on the ascending order according to fitness, tag the $R$ best-sorted moths as the initial flame population; else sort the current moth population on the ascending order according to fitness, merge the current sorted moth population into the current flame population, mark the $R$ best-sorted moths as the next flame population.

Step 6: For each moth, firstly, update its position $M_{i}$, amend the position $M_{i}$ within the lower boundary $l b$ and the upper boundary $u b$; secondly, calculate its vertical crossover solution $M v c_{i}$ through carrying out vertical crossover search, amend the position $M_{i}$ within the lower boundary $l b$ and the upper boundary $u b$.

Step 7: Therefore, the vertical crossover solutions compose the vertical moth population $M v c$, evaluate the moth population $M$ and the vertical moth population $M v c$, choose the best $N$ agents from the moth population and the vertical moth population as the current moth population, $F E s=F E s+2 N$.

Step 8: Perform the horizontal crossover search to form the horizontal moth population $M h c$, evaluate the horizontal moth population $M h c$, select the best $N$ individuals from the moth population, and the horizontal moth population as the current moth population, $F E s=$ $F E s+N$.

Step 9: If $\operatorname{rand}()<=J r$, in this work $J r=0.7$ determined by the compared test, execute the GOBL-based population optimization on the current moth population, $F E s=F E s+N, t=t+1$; else $t=t+1$.

Step 10: If $F E s<M a x F E s$, go to Step 5; else, return the best flame, end.

The flowchart of GCMFO is demonstrated in the supplementary material.

\subsection{The analysis of computational time complexity}

As it is described in the above details, the proposed GCMFO is formed by basic MFO, CC, and QOBL strategy, so the computational complexity of GCMFO is defined by the computational complexity of the original MFO, CC, and QOBL.

In the initialization stage, the random initialization consumes $N$ fitness calculations. Therefore, its computational complexity is $O(N * D)$; due to the GOBL-based population initialization strategy generates new $N$ solutions, the GOBL-based population initialization expends $N$ fitness evaluations too, and its computational complexity is $O(N * D)$ too, so in the initialization stage it 
uses $2 * N$ fitness calculations and its total computational complexity is $O(2 * N * D)$.

In every iteration, the position update mechanism of MFO pays $N$ fitness evaluations, and its computational complexity is $O(N * D)$; on the vertical crossover search, it has the same $N$ fitness evaluations and the same computational complexity $O(N * D)$ as the position update mechanism in MFO; on the horizontal crossover search, it uses $N$ fitness evaluations, and its computational complexity is $O(N * D)$ too; under the worst condition, GOBL-based population optimization operation consumes $N$ fitness calculations and its computational complexity is $O(N * D)$. So, it pays $4 * \mathrm{~N}$ fitness evaluations in each iteration, and the total computational complexity is $O(4 * N * D)$.

Therefore under the constraint of the maximum fitness evaluations MaxFEs, the total iteration numbers are $1+\left(\operatorname{MaxFEs}^{-}-2 * N\right) /(4 * N)$, so the total computational complexity is $O(2 * N * D+(\operatorname{MaxFES}-2 * N) * D)$, it can be summarized as $O(M a x F E s * D)$, so it can be concluded that the computational complexity of our method is only related to the maximum fitness evaluations MaxFEs and the dimension $D$ of the given problem.

\section{Experimental results and discussions}

In this part, a series of experiments are organized and implemented to reveal the performance of GCMFO. Firstly, the comprehensive analysis and comparison of GCMFO are performed on the CEC2017 30D test in comparison to state-of-the-art algorithms in the subsection of section 4.1. At last, in section 4.2, the proposed GCMFO is integrated into KELM to deal with real medical diagnosis cases.

In these tests, both the Wilcoxon singed-rank test and Friedman test are used to compare the results obtained by the different algorithms following the suggestion in Ref. [86, 87]. The Wilcoxon singed-rank test ${ }^{[87]}$ is adopted with a 0.05 significance level to determine whether there is a statistical significance between GCMFO and other peers. The Friedman test is also used to measure the performance differences between the GCMFO and other compared methods.

\subsection{Experiment 1: CEC2017 benchmark set test}

The CEC2017 benchmark set is adopted to evaluate the performance of GCMFO compared to state-of-the-art methods in this section; the details of the CEC2017 benchmark set can be seen in the corresponding technique report ${ }^{[88]}$ and in the supplementary material. In this experiment, fifteen typical improved swarm intelligence variants are chosen as compared algorithms; the parameter settings of these compared algorithms are illustrated in the supplementary material. To make it fair, for all the involved algorithms, the population size $N$ is set to 40, all the involved algorithms execute 30 times independently, the maximum fitness evaluations MaxFEs is set to 300000; in this test, the dimension of the given problem $D$ is 30 .

\subsubsection{Comparison with improved MFO variants}

The contrast experiment is conducted between GCMFO and other advanced MFO variants in this section. The overall ranking of all the selected MFO variants for the CEC 2017 benchmark 30D test based on average fitness results and the $p$-value results of GCMFO against other MFO variants for the CEC 2017 benchmark $30 D$ test are demonstrated in the supplementary material; it can be found that GCMFO is the No.1 peer in the competition, based on these results it can be seen that GCMFO obtains the best score 2.0 and ranks first. It can be observed that there is a significant difference between the GCMFO and other MFO variants for most of the test functions. The last line of the table indicates the comparison results between GCMFO and other MFO variants based on the Wilcoxon singed-rank test, and it can conclude that GCMFO stays ahead of other MFO variants. LGCMFO is next to the winner, GCMFO; in the competition between GCMFO and LGCMFO, GCMFO wins on 18 test functions, it loses to LGCMFO 3 functions, they get a draw on 9 functions. CMFO shows the worst performance in the competition, it is beaten by GCMFO on 28 functions, it draws with GCMFO on 2 functions.

The ranking results of all the MFO variants for the CEC 2017 benchmark 30D test based on the Friedman test are listed in Table 1. It can be observed that GCMFO gets the best score of 2.2789 on the $30 D$ test from Table 1. The Friedman ranking scores acquired by all the other competitors are more than 2.9. These results also prove the significance of the proposed GCMFO in solving the 
Table 1 The average Friedman ranking of MFO variants

\begin{tabular}{ccc}
\hline Algorithm & Friedman ranking & Rank \\
\hline CLSGMFO & 3.1889 & 3 \\
LCMFO & 2.9189 & 2 \\
QSMFO & 3.2556 & 4 \\
WEMFO & 4.2411 & 5 \\
CMFO & 5.1167 & 6 \\
GCMFO & $\mathbf{2 . 2 7 8 9}$ & $\mathbf{1}$ \\
\hline
\end{tabular}
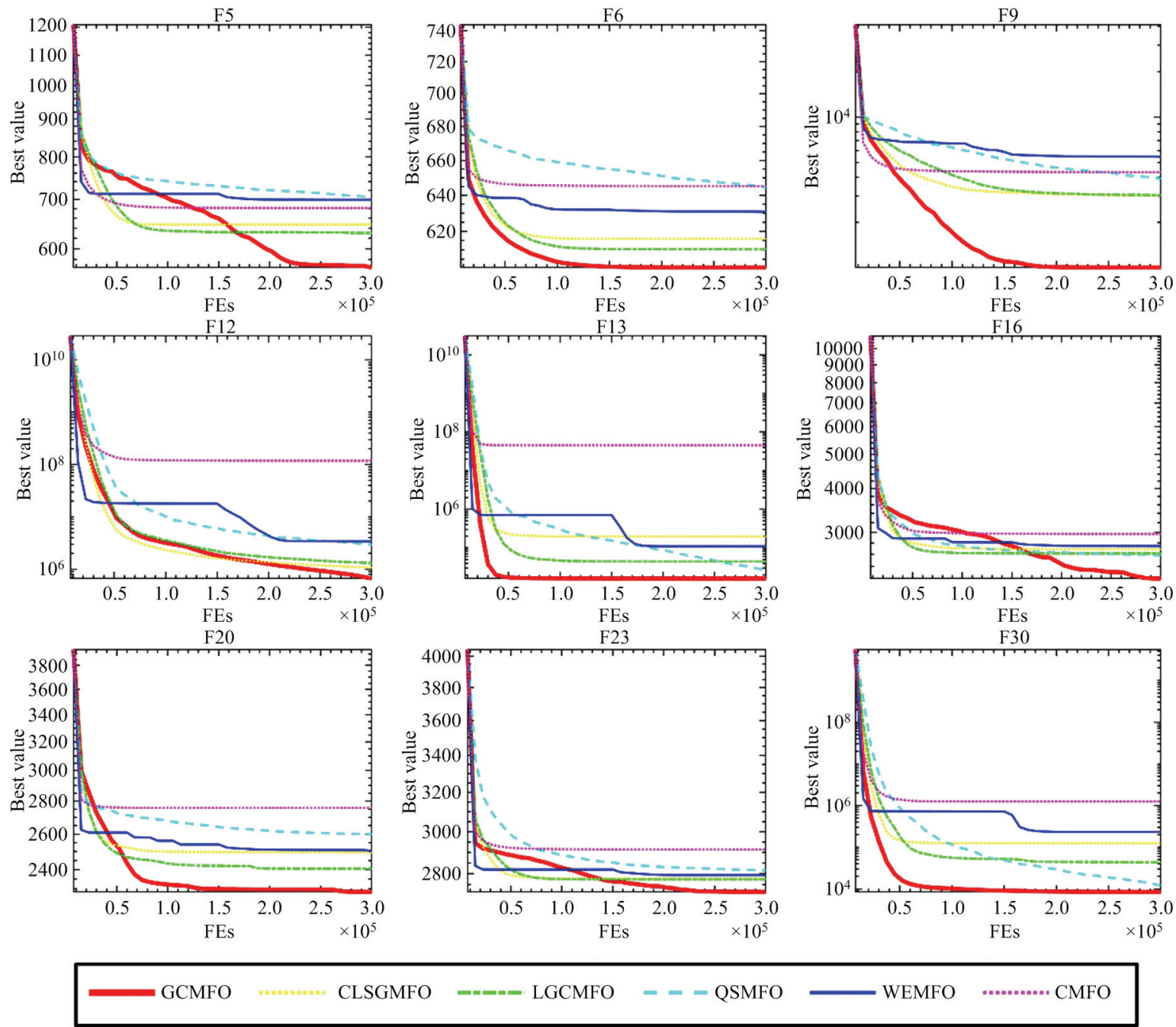

Fig. 1 The convergence plots of F5, F6, F9, F12, F13, F16, F20, F23, F30.

global optimization problem. Based on the Wilcoxon singed-rank test results and the Friedman test, it can conclude that the GOBL and CC mechanisms enhance the exploration and exploitation ability much better and result in a faster convergence speed and a better convergence accuracy, as shown in Fig. 1. Though CLSGMFO and LGCMFO embedded more strategies than the proposed GCMFO, they still perform worse than GCMFO. All these conclusions indicate that GOBL and $\mathrm{CC}$ strategy can integrate with the original MFO more reasonably.

\subsubsection{Comparison with state-of-the-art algorithms}

The overall ranking of all the methods for the CEC 2017 benchmark $30 D$ test based on average fitness results and the $p$-value results of GCMFO against other 
compared methods for the CEC 2017 benchmark 30D test are illustrated in the supplementary material. From these results, it can be known that GCMFO is on the top of the rank, the last two lines show the average ranking value and the rank value, from these results it can be found GCMFO gets the lowest score 2.2333 and the best ranking No.1. It can be observed that there is a significant difference between the GCMFO and the other methods for most of the test functions. The last line of the table reveals the comparison results between GCMFO and other competitors based on the Wilcoxon singed-rank test; it can be concluded that GCMFO is superior to each compared method. ALCPSO falls behind the winner GCMFO; in the competition between GCMFO and ALCPSO, GCMFO wins on 19 test functions, it loses to ALCPSO on 3 functions, they get a draw on 8 functions. SCADE and OBSCA perform worst in the competition; they all be beaten by GCMFO on all 30 functions.

The ranking results of all the involved algorithms for the CEC 2017 benchmark $30 D$ test based on the Friedman test are demonstrated in Table 2. It can be observed that GCMFO gains the least score of 2.6522 on the $30 \mathrm{D}$ test from Table 2 . The Friedman ranking scores gained by all the other competitors are far bigger than 3.4. These results also prove the significance of the proposed GCMFO in solving the global optimization problem. The main reason for the enhanced performance of GCMFO in comparison with the competitors can be attributed as below: first of all, GOBL can increase the population diversity and expand the search area in the search process, it can avoid the stagnation condition to take place and move to a better region, it makes the convergence curve keep a decreasing trend, so the convergence accuracy will be improved as shown in Fig. 2; then, $\mathrm{CC}$ can enhance the exploration ability of GCMFO, so the convergence rate and accuracy will be boosted, Fig. 2 can also verify this. The cooperation between GOBL and CC can make the proposed GCMFO achieve a more reasonable balance between exploration and exploitation drifts. The above results analysis and discussion confirm that GOBL and CC boost the performance of GCMFO in solving optimization problems from all angles.

\subsection{Experiment 2: Evolving KELM for medical diag nosis}

Many problems have verified that the two key parameters have a big impact on the performance of Kernel Extreme Learning Machine classifier (KELM) ${ }^{[89]}$. For example, prediction of entrepreneurial intention of Students ${ }^{[23]}$, evaluation of Sino foreign cooperative education project ${ }^{[90]}$, medical diagnosis ${ }^{[3,64,91-94]}$, second major prediction $^{[22]}$, financial stress prediction ${ }^{[95,96]}$, recognition of foreign fibers in cotton ${ }^{[97]}$.

In this section, the proposed GCMFO is applied to evolve the two parameters $C$ and $\gamma$ in KELM and then integrate GCMFO with KELM called GCMFO-KELM for medical diagnosis. To verify the performance of GCMFO-KELM, four classical classifiers consisting of $\mathrm{K}$-earest neighbor $(\mathrm{KNN})$, classification and regression tree (CART), Back Propagation (BP), Support Vector Machine (SVM), and four population-based KELM models, including WOA based KELM (WOA-KELM),

Table 2 The average Friedman ranking value gained by the algorithms

\begin{tabular}{|c|c|c|}
\hline Algorithm & Friedman ranking & Rank \\
\hline ALCPSO & 3.4156 & 2 \\
\hline CGPSO & 6.6044 & 8 \\
\hline $\mathrm{RCBA}$ & 6.4789 & 7 \\
\hline $\mathrm{CBA}$ & 7.1967 & 9 \\
\hline OBSCA & 9.0556 & 10 \\
\hline SCADE & 9.6189 & 11 \\
\hline IWOA & 4.8200 & 4 \\
\hline LWOA & 6.0211 & 6 \\
\hline IGWO & 4.5544 & 3 \\
\hline OBLGWO & 5.5822 & 5 \\
\hline GCMFO & 2.6522 & 1 \\
\hline
\end{tabular}



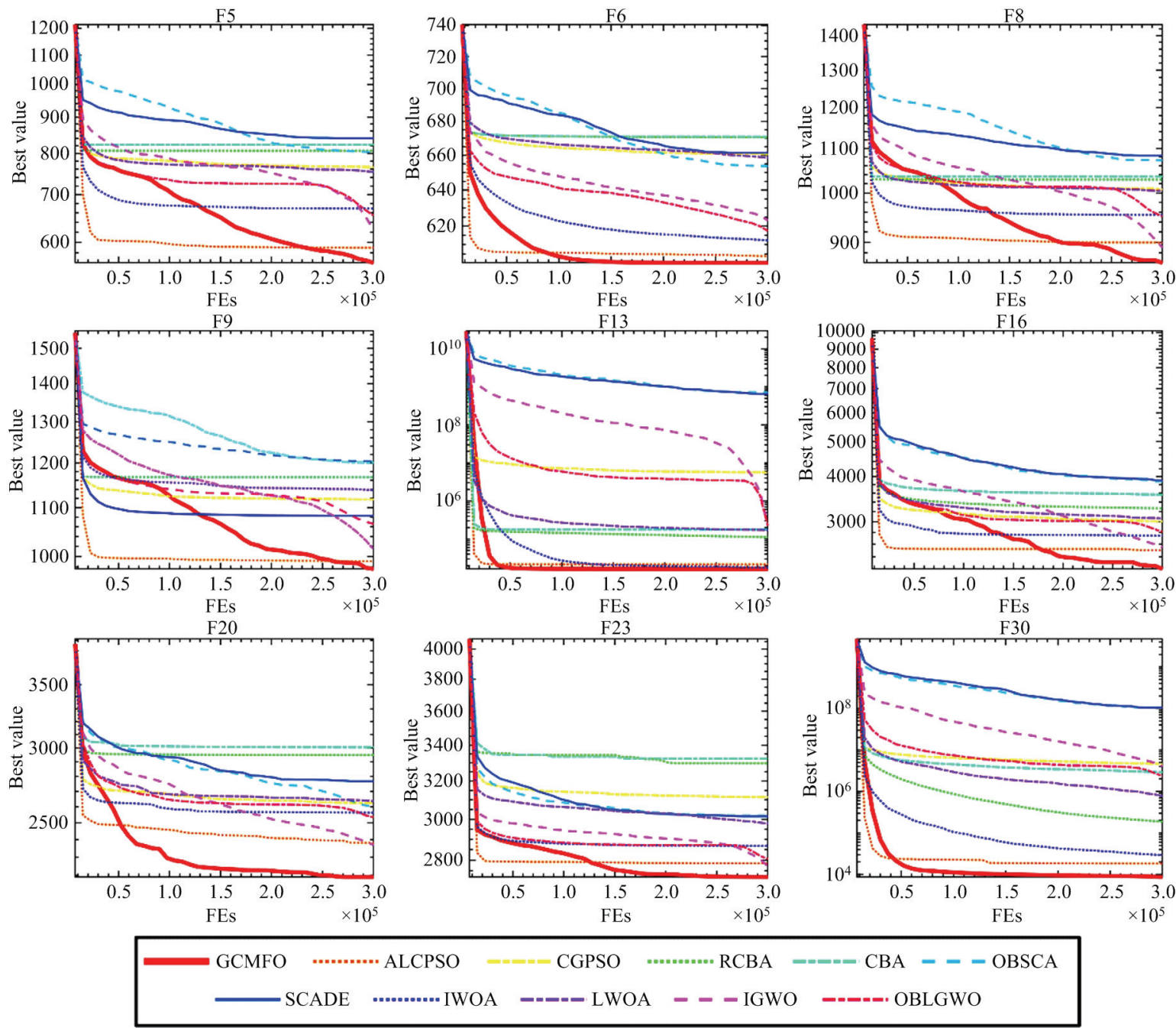

Fig. 2 The convergence curves of F5, F6, F8, F9, F13, F16, F20, F23, F30.

GWO based KELM (GWO-KELM), MFO based KELM (MFO-KELM), SSA based KELM (SSA-KELM) are adopted as compared models. All nine models are evaluated on three real medical datasets: overweight statuses and ultrasound-based thyroid cancer. In addition, there are also many other classifiers such as extreme learning machine (ELM ${ }^{[98]}$, Convolutional Neural Networks $(\mathrm{CNN})^{[99,100]}$, and so on in the classification fields.

In this experiment, to be fair, the search range of parameters $C$ and $\gamma$ in WOA-KELM, GWO-KELM, MFO-KELM, SSA-KELM, and GCMFO-KELM is $\left[2^{-8}\right.$, $\left.2^{8}\right]$, the population size is 10 , the maximum iteration number is 50 . In KNN, the value of the nearest neighbor is 1; it uses the Euclidean distance metric. In SVM, it adopts the Gaussian kernel function; both the publish coefficient and the kernel function parameter are determined by the grid search, the implementation of SVM is based on LIBSVM ${ }^{[101]}$, the domain of parameter $C$ and $\gamma$ is same as other population based KELM classifiers. In CART, the parameters are set as the default value in the software package, it is implemented by the classification tree (fitctree) algorithm in the MATLAB statistics toolbox. In BP, eight hidden neurons are utilized for the train, the maximum iteration number is 200 , the learning rate is set to 0.01 , and the mean squared error goal is set as 0.001 , the implementation of BP has based BP on the Levenberg-Marquardt training algorithm in the MATLAB neural network toolbox. To ensure the classification results be unbiased, 10-fold cross-validation will be used to evaluate the effect, in the 10-fold cross-validation, the datasets are divided into ten por- 
tions, in which nine parts are used for training, and one part is used for verification.

\subsubsection{Measure metrics for performance evaluation}

In the medical disease prediction problem, four evaluation indicators that classification accuracy (ACC), sensitivity, specificity, and Mathew Correlation Coefficient (MCC) are used to estimate the performance of the model.

ACC denotes the classification precision of the model for true positive samples and true negative samples; sensitivity indicates the classification accuracy of the true positive instances in the positive instances; specificity means the classification accuracy of the true negative instances in the negative instances; MCC denotes the reliability of the model.

\subsubsection{Appendicitis diagnosis}

This research contains the patients undergoing appendectomy in Wenzhou Central Hospital, Zhejiang Province, China from January 1, 2015, to December 31, 2016. The filtering criteria are histological reports of appendicitis. The pathology reports of the normal appendix or malignant tumor will be removed. We analyzed the medical records of the patients who met the criterion. The thesis committee approved Wenzhou Central Hospital's research and received written consent from all the patients. We have not only collected the basic demographic data such as sex, age, and vital signs containing body temperature and heart rate and also gathered the presurgical laboratory characteristics comprising white blood cells, lymphocytes, neutrophils, monocytes, eosinophils, hemoglobin, red blood cells, platelets, urea nitrogen, blood glucose, creatinine, bilirubin and $\mathrm{C}$-reactive protein (CRP). All the patients are divided into the CAP group and the UAP group. CAP includes gangrene or perforated appendicitis and/or diffuse peritonitis. Acute appendicitis includes complex and non-complicated; the criteria are operation report and pathology results of the appendix. The finding in operation is crucial when surgical results are not consistent with pathology reports.

We used SPSS 16.0 software to perform statistical analysis and adopted the Student t-test to measure the data; we also utilized Fisher's exact test to classify var- iables. There is statistical significance when the $p$-value is less than 0.05 . There are significant differences between the CAP and UAP groups on CRP, body temperature, heart rate, white blood cells, lymphocytes, neutrophils, eosinophils, urea nitrogen, and blood glucose. On these criteria, the CRP group is markedly higher than the UAP group. But there are no significant differences between the CAP group and UAP group on sex, monocytes, hemoglobin, red blood cells, platelets, creatinine, and bilirubin.

The specific results gained by different classifiers on appendicitis diagnosis are listed in Table 3. It can be observed that GCMFO-KELM gets the average value of $76.84 \%$ ACC, $77.96 \%$ sensitivity, $76.13 \%$ specificity, and $54.09 \%$ MCC.

In Table 3, it can be found conclusions as follows: concerning the criteria ACC, the classifier GCMFO-KELM based on our method GCMFO provides the highest classification accuracy in comparison to the other compared classifiers, it also holds the second-best standard deviation (Std), SSA-KELM ranks second (74.76\%) on classification accuracy, KNN, BP, CART, WOA-KELM, GWO-KELM, and MFO-KELM are behind SSA-KELM, they are less than $74.6 \%$, at the same time, SVM offers the worst classification accuracy $68.09 \%$; in terms of the criteria sensitivity, SVM acquires the biggest mean value $83.76 \%$, all the other classifiers are no more than $78 \%$, in which our method GCMFO-KELM possessed the secondbest average value $77.96 \%$, it is more than the rest of classifiers one percentage point; Considering the indicator Specificity, the proposed method GCMFO-KELM has the best results $76.13 \%$, KNN holds the second-highest value $75.57 \%$ and follows GCMFO-KELM, SVM provides the worst result $53.19 \%$. Regarding the index MCC, our method GCMFO-KELM can still get the best result $54.09 \%$, WOA-KELM, GWO-KELM, and MFO-KELM hold the second biggest result $48.88 \%$, they are ahead of the remaining classifiers, CART is worst $46.30 \%$. In a word, the above results and discussions confirm our method GCMFO-KELM shows competitive performance in comparison with other classifiers, so it can be regarded as a promising technique for real appendicitis diagnosis. 
Xia et al:: Generalized Oppositional Moth Flame Optimization with Crossover Strategy:

An Approach for Medical Diagnosis

Table 3 The results obtained by different classifiers on appendicitis diagnosis

\begin{tabular}{|c|c|c|c|c|c|c|c|c|c|c|c|c|c|}
\hline Algorithm & Indicator & Mean & Std & $\# 1$ & $\# 2$ & $\# 3$ & $\# 4$ & $\# 5$ & $\# 6$ & $\# 7$ & $\# 8$ & $\# 9$ & $\# 10$ \\
\hline $\mathrm{KNN}$ & & 0.7416 & 0.0786 & 0.7586 & 0.9000 & 0.8667 & 0.7000 & 0.7000 & 0.7000 & 0.7241 & 0.6667 & 0.7000 & 0.7000 \\
\hline CART & & 0.7320 & 0.0573 & 0.8276 & 0.7000 & 0.7667 & 0.6667 & 0.6333 & 0.7667 & 0.7586 & 0.7667 & 0.7000 & 0.7333 \\
\hline $\mathrm{BP}$ & & 0.7353 & 0.0812 & 0.7931 & 0.8000 & 0.6000 & 0.7333 & 0.8000 & 0.7931 & 0.8333 & 0.6333 & 0.6667 & 0.7000 \\
\hline SVM & & 0.6809 & 0.0739 & 0.6552 & 0.6333 & 0.7333 & 0.8000 & 0.6000 & 0.7000 & 0.6333 & 0.6333 & 0.6207 & 0.8000 \\
\hline WOA-KELM & $\mathrm{ACC}$ & 0.7451 & 0.0802 & 0.7931 & 0.9000 & 0.8667 & 0.7000 & 0.7000 & 0.7000 & 0.7241 & 0.7000 & 0.7000 & 0.6667 \\
\hline GWO-KELM & & 0.7449 & 0.0757 & 0.7586 & 0.9000 & 0.8667 & 0.7000 & 0.7000 & 0.7000 & 0.7241 & 0.7000 & 0.7000 & 0.7000 \\
\hline MFO-KELM & & 0.7451 & 0.0721 & 0.7931 & 0.9000 & 0.8333 & 0.7000 & 0.7000 & 0.7000 & 0.7241 & 0.7000 & 0.7000 & 0.7000 \\
\hline SSA-KELM & & 0.7476 & 0.0924 & 0.8333 & 0.6552 & 0.7667 & 0.6333 & 0.7667 & 0.7000 & 0.6207 & 0.8667 & 0.8667 & 0.7667 \\
\hline GCMFO-KELM & & 0.7684 & 0.0713 & 0.7667 & 0.7000 & 0.9333 & 0.8333 & 0.7931 & 0.7667 & 0.7333 & 0.7333 & 0.7000 & 0.7241 \\
\hline $\mathrm{KNN}$ & & 0.7242 & 0.0930 & 0.6923 & 0.8750 & 0.8824 & 0.7333 & 0.6429 & 0.6667 & 0.7647 & 0.6923 & 0.6923 & 0.6000 \\
\hline CART & & 0.7254 & 0.0899 & 0.7692 & 0.6875 & 0.8824 & 0.7333 & 0.7857 & 0.6667 & 0.7647 & 0.6923 & 0.5385 & 0.7333 \\
\hline $\mathrm{BP}$ & & 0.7617 & 0.1274 & 0.8462 & 0.8571 & 0.5000 & 0.6875 & 0.8462 & 0.7857 & 0.9231 & 0.7333 & 0.6250 & 0.8125 \\
\hline SVM & & 0.8376 & 0.1810 & 0.9375 & 0.8889 & 0.7500 & 1.0000 & 0.9231 & 0.6429 & 1.0000 & 0.4286 & 0.9231 & 0.8824 \\
\hline WOA-KELM & Sensitivity & 0.7378 & 0.1049 & 0.6923 & 0.8750 & 0.9412 & 0.7333 & 0.6429 & 0.6667 & 0.7647 & 0.7692 & 0.6923 & 0.6000 \\
\hline GWO-KELM & & 0.7378 & 0.1049 & 0.6923 & 0.8750 & 0.9412 & 0.7333 & 0.6429 & 0.6667 & 0.7647 & 0.7692 & 0.6923 & 0.6000 \\
\hline MFO-KELM & & 0.7319 & 0.0933 & 0.6923 & 0.8750 & 0.8824 & 0.7333 & 0.6429 & 0.6667 & 0.7647 & 0.7692 & 0.6923 & 0.6000 \\
\hline SSA-KELM & & 0.7311 & 0.1542 & 0.8667 & 0.6923 & 0.9286 & 0.4286 & 0.7778 & 0.6667 & 0.6842 & 0.8824 & 0.8125 & 0.5714 \\
\hline GCMFO-KELM & & 0.7796 & 0.1079 & 0.8235 & 0.6875 & 0.9333 & 0.9333 & 0.8333 & 0.6250 & 0.6471 & 0.7500 & 0.8125 & 0.7500 \\
\hline KNN & & 0.7557 & 0.0906 & 0.8125 & 0.9286 & 0.8462 & 0.6667 & 0.7500 & 0.7333 & 0.6667 & 0.6471 & 0.7059 & 0.8000 \\
\hline CART & & 0.7302 & 0.1251 & 0.8750 & 0.7143 & 0.6154 & 0.6000 & 0.5000 & 0.8667 & 0.7500 & 0.8235 & 0.8235 & 0.7333 \\
\hline $\mathrm{BP}$ & & 0.7184 & 0.0909 & 0.7500 & 0.7500 & 0.7500 & 0.7857 & 0.7647 & 0.8000 & 0.7647 & 0.5333 & 0.7143 & 0.5714 \\
\hline SVM & & 0.5319 & 0.1993 & 0.3077 & 0.5238 & 0.7000 & 0.5385 & 0.3529 & 0.7500 & 0.2667 & 0.8125 & 0.3750 & 0.6923 \\
\hline WOA-KELM & Specificity & 0.7476 & 0.0912 & 0.8750 & 0.9286 & 0.7692 & 0.6667 & 0.7500 & 0.7333 & 0.6667 & 0.6471 & 0.7059 & 0.7333 \\
\hline GWO-KELM & & 0.7480 & 0.0852 & 0.8125 & 0.9286 & 0.7692 & 0.6667 & 0.7500 & 0.7333 & 0.6667 & 0.6471 & 0.7059 & 0.8000 \\
\hline MFO-KELM & & 0.7542 & 0.0924 & 0.8750 & 0.9286 & 0.7692 & 0.6667 & 0.7500 & 0.7333 & 0.6667 & 0.6471 & 0.7059 & 0.8000 \\
\hline SSA-KELM & & 0.7441 & 0.1396 & 0.8000 & 0.6250 & 0.6250 & 0.6957 & 0.7500 & 0.7333 & 0.5000 & 0.8462 & 0.9286 & 0.9375 \\
\hline GCMFO-KELM & & 0.7613 & 0.1121 & 0.6923 & 0.7143 & 0.9333 & 0.7333 & 0.7647 & 0.9286 & 0.8462 & 0.7143 & 0.5714 & 0.7143 \\
\hline KNN & & 0.4809 & 0.1567 & 0.5097 & 0.8018 & 0.7285 & 0.4009 & 0.3955 & 0.4009 & 0.4314 & 0.3363 & 0.3955 & 0.4082 \\
\hline CART & & 0.4630 & 0.1084 & 0.6505 & 0.4009 & 0.5232 & 0.3363 & 0.2958 & 0.5443 & 0.5097 & 0.5218 & 0.3805 & 0.4667 \\
\hline $\mathrm{BP}$ & & 0.4807 & 0.1563 & 0.5933 & 0.6071 & 0.2500 & 0.4732 & 0.6054 & 0.5857 & 0.6832 & 0.2722 & 0.3393 & 0.3975 \\
\hline SVM & & 0.4084 & 0.1174 & 0.3228 & 0.3860 & 0.4330 & 0.6309 & 0.3234 & 0.3955 & 0.3922 & 0.2624 & 0.3464 & 0.5909 \\
\hline WOA-KELM & $\mathrm{MCC}$ & 0.4888 & 0.1599 & 0.5815 & 0.8018 & 0.7305 & 0.4009 & 0.3955 & 0.4009 & 0.4314 & 0.4135 & 0.3955 & 0.3363 \\
\hline GWO-KELM & & 0.4888 & 0.1510 & 0.5097 & 0.8018 & 0.7305 & 0.4009 & 0.3955 & 0.4009 & 0.4314 & 0.4135 & 0.3955 & 0.4082 \\
\hline MFO-KELM & & 0.4888 & 0.1429 & 0.5815 & 0.8018 & 0.6591 & 0.4009 & 0.3955 & 0.4009 & 0.4314 & 0.4135 & 0.3955 & 0.4082 \\
\hline SSA-KELM & & 0.4795 & 0.2211 & 0.6682 & 0.3158 & 0.5731 & 0.1115 & 0.5218 & 0.4009 & 0.1805 & 0.7285 & 0.7411 & 0.5541 \\
\hline GCMFO-KELM & & 0.5409 & 0.1460 & 0.5218 & 0.4009 & 0.8667 & 0.6804 & 0.5894 & 0.5731 & 0.4932 & 0.4643 & 0.3975 & 0.4213 \\
\hline
\end{tabular}

\subsubsection{Ultrasound-based thyroid cancer diagnosis}

This research is based on 187 patients with thyroid nodules confirmed histologically and approved by Wenzhou Central Hospital relevant authorities ${ }^{[102,103]}$; at the same time the written informed consent is obtained from all subjects. The data is from January 2011 to December 2014. All the patients are checked by ultrasound-guided fine-needle aspiration biopsy. The pathological results of thyroidectomy determine the final diagnosis of benign and malignant nodules. The patients include 114 benign nodules from 106 patients (82 females and 24 males; average age 51.22 \pm 12.09$)$ and 89 malignant nodules from 81 patients ( 69 females and 12 males; average age 48.09 \pm 11.37 ). All malignant tumors are papillary carcinoma and benign lesions are nodular hyperplasia. The size of nodules is between $5 \mathrm{~mm}$ and 62

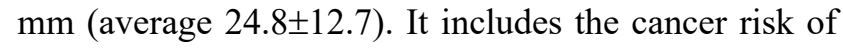
each feature. It can be found that the following features 
are related to a malignant tumor, such as taller than the wide, solid interior, irregular edge, hypoechoic, and microcalcification based on chi-square analysis, at the same time, there are some features related to benign nodule as follow: the wide of the shape is bigger than tall, no solid component, smooth edge, and isopach. There is no significant difference between some features and benign and malignant nodules.

The concrete results gained by different classifiers on ultrasound-based thyroid cancer diagnosis are shown in Table 4. It can be seen that GCMFO-KELM obtains the average value of $87.16 \%$ ACC, $77.97 \%$ sensitivity, $94.32 \%$ specificity, and $73.75 \% \mathrm{MCC}$.

Based on Table 4, it can be got conclusions as below: about the criteria ACC, our method GCMFO-KELM gains the highest classification accuracy $85.00 \%$, by comparison with the other compared classifiers, SSA-KELM comes second $(82.92 \%)$ on classification accuracy, CART, SVM, GWO-KELM, and MFO-KELM are behind SSA-KELM, the disparity

Table 4 The results acquired by different classifiers on ultrasound-based thyroid cancer diagnosis

\begin{tabular}{|c|c|c|c|c|c|c|c|c|c|c|c|c|c|}
\hline Algorithm & Indicator & Mean & Std & $\# 1$ & $\# 2$ & $\# 3$ & $\# 4$ & $\# 5$ & $\# 6$ & $\# 7$ & $\# 8$ & $\# 9$ & $\# 10$ \\
\hline KNN & & 0.7535 & 0.0927 & 0.6842 & 0.7778 & 0.8947 & 0.7368 & 0.7368 & 0.8421 & 0.8421 & 0.7778 & 0.6111 & 0.6316 \\
\hline CART & & 0.8170 & 0.1151 & 0.8947 & 0.7222 & 0.7895 & 1.0000 & 0.9474 & 0.6667 & 0.8333 & 0.7368 & 0.6842 & 0.8947 \\
\hline SVM & & 0.8132 & 0.0944 & 0.7895 & 0.8947 & 0.9444 & 0.8333 & 0.7222 & 0.6316 & 0.8421 & 0.8421 & 0.8947 & 0.7368 \\
\hline WOA-KELM & $\mathrm{ACC}$ & 0.8076 & 0.0914 & 0.7895 & 0.9474 & 0.9444 & 0.7222 & 0.7778 & 0.6842 & 0.7368 & 0.7895 & 0.8947 & 0.7895 \\
\hline MFO-KELM & & 0.8135 & 0.0829 & 0.7895 & 0.8947 & 0.9444 & 0.8333 & 0.7778 & 0.6316 & 0.8421 & 0.7895 & 0.8421 & 0.7895 \\
\hline SSA-KELM & & 0.8292 & 0.0779 & 0.7895 & 0.9474 & 0.9444 & 0.8333 & 0.7778 & 0.6842 & 0.8421 & 0.8421 & 0.8421 & 0.7895 \\
\hline GCMFO-KELM & & 0.8500 & 0.0952 & 0.8421 & 0.8947 & 0.9474 & 0.9474 & 0.6667 & 0.8889 & 0.9444 & 0.7368 & 0.8421 & 0.7895 \\
\hline $\mathrm{KNN}$ & & 0.4921 & 0.1753 & 0.2500 & 0.6250 & 0.7778 & 0.2857 & 0.5000 & 0.5714 & 0.6667 & 0.5000 & 0.4444 & 0.3000 \\
\hline WOA-KELM & Sensitivity & 0.6946 & 0.1513 & 0.5714 & 0.8333 & 0.8750 & 0.3750 & 0.7000 & 0.8333 & 0.6667 & 0.6250 & 0.8000 & 0.6667 \\
\hline GWO-KELM & & 0.7662 & 0.0977 & 0.7143 & 0.8333 & 0.8750 & 0.6250 & 0.7000 & 0.8333 & 0.7778 & 0.6250 & 0.9000 & 0.7778 \\
\hline MFO-KELM & & 0.7408 & 0.1176 & 0.5714 & 0.8333 & 0.8750 & 0.6250 & 0.7000 & 0.8333 & 0.7778 & 0.6250 & 0.9000 & 0.6667 \\
\hline SSA-KELM & & 0.7644 & 0.1062 & 0.5714 & 0.8333 & 0.8750 & 0.6250 & 0.7000 & 0.8333 & 0.7778 & 0.7500 & 0.9000 & 0.7778 \\
\hline GCMFO-KELM & & 0.7300 & 0.1336 & 0.7500 & 0.7778 & 0.8333 & 0.8571 & 0.4545 & 0.8750 & 0.8000 & 0.5714 & 0.6667 & 0.7143 \\
\hline $\mathrm{KNN}$ & & 0.9504 & 0.0745 & 1.0000 & 0.9000 & 1.0000 & 1.0000 & 0.9091 & 1.0000 & 1.0000 & 0.9167 & 0.7778 & 1.0000 \\
\hline CART & & 0.8445 & 0.2182 & 0.8333 & 1.0000 & 0.8889 & 1.0000 & 1.0000 & 0.2857 & 0.9091 & 0.7778 & 0.7500 & 1.0000 \\
\hline $\mathrm{BP}$ & & 0.8627 & 0.1365 & 0.9231 & 0.8000 & 1.0000 & 0.8333 & 1.0000 & 0.6429 & 1.0000 & 0.6364 & 0.8750 & 0.9167 \\
\hline $\mathrm{KNN}$ & & 0.5180 & 0.1709 & 0.4022 & 0.5534 & 0.8051 & 0.4491 & 0.4587 & 0.6761 & 0.7161 & 0.4725 & 0.2357 & 0.4108 \\
\hline CART & & 0.6107 & 0.2434 & 0.8051 & 0.4432 & 0.5955 & 1.0000 & 0.9000 & 0.2548 & 0.6447 & 0.4778 & 0.3214 & 0.6642 \\
\hline $\mathrm{BP}$ & & 0.6279 & 0.1683 & 0.8895 & 0.8090 & 0.6202 & 0.4200 & 0.5976 & 0.3906 & 0.4564 & 0.6513 & 0.6708 & 0.7738 \\
\hline SVM & & 0.6369 & 0.1679 & 0.5347 & 0.7564 & 0.8919 & 0.6934 & 0.4842 & 0.3500 & 0.6854 & 0.6746 & 0.8090 & 0.4893 \\
\hline WOA-KELM & $\mathrm{MCC}$ & 0.6234 & 0.1724 & 0.5347 & 0.8797 & 0.8919 & 0.5000 & 0.5750 & 0.4177 & 0.4719 & 0.5673 & 0.8090 & 0.5866 \\
\hline GWO-KELM & & 0.6437 & 0.1420 & 0.6548 & 0.7564 & 0.8919 & 0.6934 & 0.5750 & 0.3500 & 0.6854 & 0.5673 & 0.6854 & 0.5778 \\
\hline MFO-KELM & & 0.6326 & 0.1457 & 0.5347 & 0.7564 & 0.8919 & 0.6934 & 0.5750 & 0.3500 & 0.6854 & 0.5673 & 0.6854 & 0.5866 \\
\hline SSA-KELM & & 0.6616 & 0.1464 & 0.5347 & 0.8797 & 0.8919 & 0.6934 & 0.5750 & 0.4177 & 0.6854 & 0.6746 & 0.6854 & 0.5778 \\
\hline GCMFO-KELM & & 0.7114 & 0.1681 & 0.7246 & 0.8051 & 0.8797 & 0.8895 & 0.4947 & 0.7750 & 0.8619 & 0.4200 & 0.7161 & 0.5476 \\
\hline
\end{tabular}


between them and SSA-KELM is not bigger, at the same time, KNN provides the worst classification accuracy $75.35 \%$; In terms of the criteria sensitivity, GWO-KELM possesses the best result $77.97 \%$, all the other classifiers are no more than $76.5 \%$, among them SSA-KELM obtain the second-best result $76.44 \%$, it is better than the remaining classifiers, our method GCMFO-KELM is better than KNN, CART, SVM, and WOA-KELM, but it is worse than BP, MFO-KELM. $\mathrm{KNN}$ is the worst $49.21 \%$; Respecting the indicator Specificity, our method GCMFO-KELM provides the best results $95.67 \%$ and the minimal Std $0.0721, \mathrm{KNN}$ gets the second-best result $95.04 \%$, CART has the worst result $84.45 \%$. Considering the criteria MCC, our method GCMFO-KELM can still provide the best result $71.14 \%$, SSA-KELM has the second-best result $66.16 \%$, it is bigger than the rest classifiers, among them $\mathrm{KNN}$ is worst $51.80 \%$. In brief, the results and discussions approve that our method GCMFO-KELM performs best compared to other classifiers, so it can be considered a promising scheme for real ultrasound-based thyroid cancer diagnosis.

In the future work, GCMFO can be a potential tool for face recognition and micro-expression recognition $^{[104,105]}$, disease diagnosis ${ }^{[106-111]}$, secure encryption $^{[112]}$, geometric modeling ${ }^{[113]}$, operating condition identification in aluminium electrolysis ${ }^{[114]}$, image editing $^{[115-117]}$, gold price prediction ${ }^{[118]}$, cluster analysis $^{[119-121]}$, image recognition ${ }^{[122,123]}$, service ecosystem $^{[124]}$, prediction and improvement of brain behavior $^{[125,126]}$, shape registration ${ }^{[127]}$, and lunar impact crater identification and age estimation ${ }^{[128]}$, renewable energy generation $^{[129]}$, feature selection ${ }^{[130-133]}$, and image dehazing $^{[134-137]}$.

\section{Conclusions and future works}

In this paper, an enhanced MFO named GCMFO is proposed by introducing GOBL and $\mathrm{CC}$ mechanisms into the original MFO. Both GOBL and CC mechanisms cooperate to boost the exploration and exploitation of MFO. The proposed GCMFO is verified by solving the benchmark optimization problems, and the parameter optimization of KELM for solving real-life medical diagnosis cases. The experimental results reveal that the presented GCMFO can effectively solve practical opti- mization problems and be treated as a promising solution for real-world optimization problems.

In the future, we will focus our research on the following aspects: On the one hand, GCMFO can be equipped with high-performance techniques to reduce the computational burden; on the other hand, GCMFO can be modified to the multi-objective version and binary version for specific application.

\section{Acknowledgment}

This research is supported by the National Natural Science Foundation of China (62076185, U1809209), Zhejiang Provincial Natural Science Foundation of China (LY21F020030), Wenzhou Science \& Technology Bureau (2018ZG016), Taif University Researchers Supporting Project Number (TURSP-2020/125), Taif University, Taif, Saudi Arabia. We thank Doctor Ali Asghar Heidari for his effort on polishing the paper. We acknowledge the efforts of the pioneer editor and valuable comments of anonymous reviewers during the revision of this research.

* All supplementary materials are available at https://doi.org/10.1007/s42235-021-0068-1.

Open Access This article is licensed under a Creative Commons Attribution 4.0 International License, which permits use, sharing, adaptation, distribution and reproduction in any medium or format, as long as you give appropriate credit to the original author(s) and the source, provide a link to the Creative Commons licence, and indicate if changes were made.

The images or other third party material in this article are included in the article's Creative Commons licence, unless indicated otherwise in a credit line to the material. If material is not included in the article's Creative Commons licence and your intended use is not permitted by statutory regulation or exceeds the permitted use, you will need to obtain permission directly from the copyright holder.

To view a copy of this licence, visit http://creativecommons.org/licenses/by/4.0/.

\section{References}

[1] Ahmadianfar L, Heidari A A, Gandomi A H, Chu X F, Chen H L. RUN beyond the metaphor: An efficient optimization 
algorithm based on Runge Kutta method. Expert Systems with Applications, 2021, 181, 115079.

[2] Yang Y L, Chen H L, Heidari A A, Gandomi A H. Hunger games search: Visions, conception, implementation, deep analysis, perspectives, and towards performance shifts. Expert Systems with Applications, 2021, 177, 114864.

[3] Wang M J, Chen H L, Yang B, Zhao X H, Hu L F, Cai Z N, Huang H, Tong C F. Toward an optimal kernel extreme learning machine using a chaotic moth-flame optimization strategy with applications in medical diagnoses. Neurocomputing, 2017, 267, 69-84.

[4] Zhao X H, Zhang X, Cai Z N, Tian X, Wang X Q, Huang Y, Chen H L, Hu L F. Chaos enhanced grey wolf optimization wrapped ELM for diagnosis of paraquat-poisoned patients. Computational Biology and Chemistry, 2019, 78, 481-490.

[5] Wang M J, Chen H L. Chaotic multi-swarm whale optimizer boosted support vector machine for medical diagnosis. Applied Soft Computing, 2020, 88, 105946.

[6] Shan W F, Qiao Z L, Heidari A A, Chen H L, Turabieh H, Teng Y T. Double adaptive weights for stabilization of moth flame optimizer: Balance analysis, engineering cases, and medical diagnosis. Knowledge-Based Systems, 2021, 214, 106728.

[7] Yu C Y, Chen M X, Cheng K, Zhao X H, Ma C, Kuang F J, Chen H L. SGOA: Annealing-behaved grasshopper optimizer for global tasks. Engineering with Computers, 2021, https://doi.org/10.1007/s00366-020-01234-1.

[8] Zhang Y N, Liu R J, Heidari A A, Wang X, Chen Y, Wang M J, Chen H L. Towards augmented kernel extreme learning models for bankruptcy prediction: Algorithmic behavior and comprehensive analysis. Neurocomputing, 2021, 430, 185-212.

[9] Wang M J, Chen H L, Li H Z, Cai Z N, Zhao X H, Tong C F, Li J, Xu X. Grey wolf optimization evolving kernel extreme learning machine: Application to bankruptcy prediction. Engineering Applications of Artificial Intelligence, 2017, 63, 54-68.

[10] Jiao S, Chong G S, Huang C C, Hu H Q, Wang M J, Heidari A A, Chen H L, and Zhao X H. Orthogonally adapted Harris hawks optimization for parameter estimation of photovoltaic models. Energy, 2020, 203, 117804.

[11] Zhang H L, Heidari A A, Wang M J, Zhang L J, Chen H L, Li C Y. Orthogonal Nelder-Mead moth flame method for parameters identification of photovoltaic modules. Energy Conversion and Management, 2020, 211, 112764.

[12] Chen H L, Jiao S, Heidari A A, Wang M J, Chen X, Zhao X
H. An opposition-based sine cosine approach with local search for parameter estimation of photovoltaic models. Energy Conversion and Management, 2019, 195, 927-942.

[13] Tu J Z, Chen H L, Liu J C, Heidari A A, Zhang X Q, Wang M J, Ruby R, Pham Q V. Evolutionary biogeography-based whale optimization methods with communication structure: Towards measuring the balance. Knowledge-Based Systems, 2021, 212, 106642.

[14] Song S M, Wang P J, Heidari A A, Wang M J, Zhao X H, Chen H L, He W M, Xu S L. Dimension decided Harris hawks optimization with Gaussian mutation: Balance analysis and diversity patterns. Knowledge-Based Systems, 2021, 215, 106425.

[15] Yu H L, Li W S, Chen C C, Liang J, Gui W Y, Wang M J, Chen H L. Dynamic Gaussian bare-bones fruit fly optimizers with abandonment mechanism: Method and analysis. Engineering with Computers, 2020, https://doi.org/10.1007/s00366-020-01174-w.

[16] Wang X Y, Chen H L, Heidari A A, Zhang X, Xu J, Xu Y T, Huang H. Multi-population following behavior-driven fruit fly optimization: A Markov chain convergence proof and comprehensive analysis. Knowledge-Based Systems, 2020, 210, 106437.

[17] Liang X, Cai Z N, Wang M J, Zhao X H, Chen H L, Li C Y. Chaotic oppositional sine-cosine method for solving global optimization problems. Engineering with Computers, 2020, https://doi.org/10.1007/s00366-020-01083-y.

[18] Zhang H L, Cai Z N, Ye X J, Wang M J, Kuang F J, Chen H L, Li C Y, Li Y P. A multi-strategy enhanced salp swarm algorithm for global optimization. Engineering with Computers, 2020, https://doi.org/10.1007/s00366020-01099-4.

[19] Ba A F, Huang H, Wang M J, Ye X J, Gu Z Y, Chen H L, Cai $X$ D. Levy-based antlion-inspired optimizers with orthogonal learning scheme. Engineering with Computers, 2020, https://doi.org/10.1007/s00366-020-01042-7.

[20] Fan Y, Wang P J, Mafarja M, Wang M J, Zhao X H, Chen H L. A bioinformatic variant fruit fly optimizer for tackling optimization problems. Knowledge-Based Systems, 2021, 213, 106704.

[21] Zhang X, Xu Y T, Yu C Y, Heidari A A, Li S M, Chen H L, Li C Y. Gaussian mutational chaotic fruit fly-built optimization and feature selection. Expert Systems with Applications, 2020, 141, 112976.

[22] Cai Z N, Gu J H, Luo J, Zhang Q, Chen H L, Pan Z F, Li Y P, Li C Y. Evolving an optimal kernel extreme learning machine by using an enhanced grey wolf optimization 
strategy. Expert Systems with Applications, 2019, 138, 112814 .

[23] Wei Y, Lv H J, Chen M X, Wang M J, Heidari A A, Chen H L, Li C Y. Predicting entrepreneurial intention of students: An extreme learning machine with Gaussian Barebone Harris Hawks optimizer. IEEE Access, 2020, 8, 76841-76855.

[24] Zhu W, Ma C, Zhao X H, Wang M J, Heidari A A, Chen H L, Li C Y. Evaluation of sino foreign cooperative education project using orthogonal sine cosine optimized kernel extreme learning machine. IEEE Access, 2020, 8, 61107-61123.

[25] Zeng G Q, Lu Y Z, Mao W J. Modified extremal optimization for the hard maximum satisfiability problem. Journal of Zhejiang University-Science C-Computers \& Electronics, 2011, 12, 589-596.

[26] Zeng G Q, Lu Y Z, Dai Y X, Wu Z G, Mao W J, Zhang Z J, Zheng $\mathrm{C} \mathrm{W}$. Backbone guided extremal optimization for the hard maximum satisfiability problem. International Journal of Innovative Computing Information and Control, 2012, 8, 8355-8366.

[27] Zeng G Q, Lu K D, Dai Y X, Zhang Z J, Chen M R, Zheng C W, Wu D, Peng W W. Binary-coded extremal optimization for the design of PID controllers. Neurocomputing, 2014, 138, 180-188.

[28] Zeng G Q, Chen J, Dai Y X, Li L M, Zheng C W, Chen M R. Design of fractional order PID controller for automatic regulator voltage system based on multi-objective extremal optimization. Neurocomputing, 2015, 160, 173-184.

[29] Zeng G Q, Xie X Q, Chen M R, Weng J. Adaptive population extremal optimization-based PID neural network for multivariable nonlinear control systems. Swarm and Evolutionary Computation, 2019, 44, 320-334.

[30] Chen M R, Zeng G Q, Lu K D, Weng J. A two-layer nonlinear combination method for short-term wind speed prediction based on ELM, ENN, and LSTM. IEEE Internet of Things Journal, 2019, 6, 6997-7010.

[31] Deng W, Liu H L, Xu J J, Zhao H M, Song Y J. An improved quantum-inspired differential evolution algorithm for deep belief network. IEEE Transactions on Instrumentation and Measurement, 2020, 69, 7319-7327.

[32] Zhao H M, Liu H D, Xu J J, Deng W. Performance prediction using high-order differential mathematical morphology gradient spectrum entropy and extreme learning machine. IEEE Transactions on Instrumentation \& Measurement, 2019, 4165-4172.

[33] Deng W, Xu J J, Song Y J, Zhao H M. An effective improved co-evolution ant colony optimization algorithm with multi-strategies and its application. International Journal of Bio-Inspired Computation, 2020, 16, 158-170.

[34] Deng W, Xu J J, Zhao H M, Song Y J. A novel gate resource allocation method using improved PSO-based QEA. IEEE Transactions on Intelligent Transportation Systems, 2020, https://doi.org/10.1109/TITS.2020.3025796.

[35] Pang J H, Zhou H M, Tsai Y C, Chou F D. A scatter simulated annealing algorithm for the bi-objective scheduling problem for the wet station of semiconductor manufacturing. Computers \& Industrial Engineering, 2018, 123, 54-66.

[36] Zhou H M, Pang J H, Chen P K, Chou F D. A modified particle swarm optimization algorithm for a batch-processing machine scheduling problem with arbitrary release times and non-identical job sizes. Computers \& Industrial Engineering, 2018, 123, 67-81.

[37] Kennedy J, Eberhart R. Particle swarm optimization. Proceedings of ICNN'95 - International Conference on Neural Networks, Perth, WA, Australia, 1995, 1942-1948.

[38] Karaboga D. An Idea Based on Honey Bee Swarm for Numerical Optimization, techinical report-TR06, Erciyes University, 2005, Turkey.

[39] Mirjalili S. Moth-flame optimization algorithm: A novel nature-inspired heuristic paradigm. Knowledge-Based Systems, 2015, 89, 228-249.

[40] Heidari A A, Mirjalili S, Faris H, Aljarah I, Mafarja M, Chen H L. Harris hawks optimization: Algorithm and applications. Future Generation Computer Systems - the International Journal of Escience, 2019, 97, 849-872.

[41] Li S M, Chen H L, Wang M J, Heidari A A, Mirjalili S. Slime mould algorithm: A new method for stochastic optimization. Future Generation Computer Systems-the International Journal of Escience, 2020, 111, 300-323.

[42] Sapre S, Mini S. Opposition-based moth flame optimization with Cauchy mutation and evolutionary boundary constraint handling for global optimization. Soft Computing, 2018, 23, 6023-6041.

[43] Emary E, Zawbaa H M. Impact of chaos functions on modern swarm optimizers. PLoS ONE, 2016, 11, e0158738.

[44] Guvenc U, Duman S, Hinıslioglu Y. Chaotic moth swarm algorithm. IEEE International Conference on INnovations in Intelligent SysTems and Applications (INISTA), Gdynia, Poland, 2017, 90-95.

[45] Xu Y T, Chen H L, Heidari A A, Luo J, Zhang Q, Zhao X H, 
Li C Y. An efficient chaotic mutative moth-flame-inspired optimizer for global optimization tasks. Expert Systems with Applications, 2019, 129, 135-155.

[46] Li Z M, Zhou Y Q, Zhang S, Song J M. Levy-flight moth-flame algorithm for function optimization and engineering design problems. Mathematical Problems in Engineering, 2016, 2016, https://doi.org/10.1155/ 2016/1423930.

[47] Xu Y T, Chen H L, Luo J, Zhang Q, Jiao S, Zhang X Q. Enhanced Moth-flame optimizer with mutation strategy for global optimization. Information Sciences, 2019, 492, 181-203.

[48] Elsakaan A A, El-Sehiemy R A, Kaddah S S, Elsaid M I. An enhanced moth-flame optimizer for solving non-smooth economic dispatch problems with emissions. Energy, 2018, 157, 1063-1078.

[49] Zhang H L, Li R, Cai Z N, Gu Z Y, Heidari A A, Wang M J, Chen H L, Chen M Y. Advanced orthogonal moth flame optimization with Broyden-Fletcher-Goldfarb-Shanno algorithm: Framework and real-world problems. Expert Systems with Applications, 2020, 159, 113617.

[50] Bhesdadiya R H, Trivedi I N, Jangir P, Kumar A, Jangir N, Totlani R. A novel hybrid approach particle swarm optimizer with moth-flame optimizer algorithm. In Bhatia S, Mishra K, Tiwari S, Singh V (eds.), Advances in Computer and Computational Sciences. Advances in Intelligent Systems and Computing, Springer, Singapore, 2017, 553, 569-577.

[51] Khalilpourazari S, Khalilpourazary S. An efficient hybrid algorithm based on Water Cycle and Moth-Flame Optimization algorithms for solving numerical and constrained engineering optimization problems. Soft Computing, 2019, 23, 1699-1722.

[52] Khalilpourazari S, Pasandideh S L R. Modeling and optimization of multi-item multi-constrained EOQ model for growing items. Knowledge-Based Systems, 2019, 164, $150-162$.

[53] Zhang L, Mistry K, Neoh S C, Lim C P. Intelligent facial emotion recognition using moth-firefly optimization. Knowledge-Based Systems, 2016, 111, 248-267.

[54] Zhao X D, Fang Y M, Liu L, Xu M, Zhang P. Ameliorated moth-flame algorithm and its application for modeling of silicon content in liquid iron of blast furnace based fast learning network. Applied Soft Computing, 2020, 94, 106418

[55] Chen C C, Wang X C, Yu H L, Wang M J, Chen H L. Dealing with multi-modality using synthesis of moth-flame optimizer with sine cosine mechanisms. Mathematics and Computers in Simulation, 2021, 188, 291-318.

[56] K S R, Panwar L K, Panigrahi B K, Kumar R. Solution to unit commitment in power system operation planning using binary coded modified moth flame optimization algorithm (BMMFOA): A flame selection based computational technique. Journal of Computational Science, 2018, 25, 298-317.

[57] Punnathanam V, Kommadath R, Kotecha P. Extension and performance evaluation of recent optimization techniques on mixed integer optimization problems. IEEE Congress on Evolutionary Computation, Vancouver, BC, Canada, 2016, 4390-4397.

[58] Savsani V, Tawhid M A. Non-dominated sorting moth flame optimization (NS-MFO) for multi-objective problems. Engineering Applications of Artificial Intelligence, 2017, 63, $20-32$.

[59] Dubey H M, Pandit M, Panigrahi B K. An overview and comparative analysis of recent bio-inspired optimization techniques for wind integrated multi-objective power dispatch. Swarm and Evolutionary Computation, 2018, 38, 12-34.

[60] Sayed G I, Hassanien A E. Moth-flame swarm optimization with neutrosophic sets for automatic mitosis detection in breast cancer histology images. Applied Intelligence, 2017, 47, 397-408.

[61] Li J F, Niu D X, Wu M, Wang Y L, Li F, Dong H R. Research on battery energy storage as backup power in the operation optimization of a regional Integrated energy system. Energies, 2018, 11, 2990-3009.

[62] Li C B, Li S K, Liu Y Q. A least squares support vector machine model optimized by moth-flame optimization algorithm for annual power load forecasting. Applied Intelligence, 2016, 45, 1166-1178.

[63] Jiang P, Li R, Lu H, Zhang X. Modeling of electricity demand forecast for power system. Neural Computing and Applications, 2019, 32, 6857-6875.

[64] Luo J, Chen H L, Hu Z Y, Huang H, Wang P J, Wang X Q, Lv $\mathrm{X}$ E, Wen $\mathrm{C}$ C. A new kernel extreme learning machine framework for somatization disorder diagnosis. IEEE Access, 2019, 7, 45512-45525.

[65] Li C Y, Hou L X, Sharma B Y, Li H Z, Chen C S, Li Y P, Zhao X H, Huang H, Cai Z N, Chen H L. Developing a new intelligent system for the diagnosis of tuberculous pleural effusion. Computer Methods and Programs Biomedicine, 2018, 153, 211-225.

[66] Xu Y T, Yu K T, Wang P J, Chen H L, Zhao X H, Zhu J Y. A 
New hybrid machine learning approach for prediction of phenanthrene toxicity on mice. IEEE Access, 2019, 7, 138461-138472.

[67] Deng W, Xu J, Gao X Z, Zhao H. An enhanced MSIQDE algorithm with novel multiple strategies for global optimization problems. IEEE Transactions on Systems, Man, and Cybernetics: Systems, 2020, https://doi.org/ 10.1109/TSMC.2020.3030792.

[68] Song Y J, Wu D Q, Deng W, Gao X Z, Li T Y, Zhang B, Li Y G. MPPCEDE: Multi-population parallel co-evolutionary differential evolution for parameter optimization. Energy Conversion and Management, 2021, 228, 113661.

[69] Deng W, Xu J J, Song Y J, Zhao H M. Differential evolution algorithm with wavelet basis function and optimal mutation strategy for complex optimization problem. Applied Soft Computing, 2021, 100, 106724.

[70] Song Y J, Wu D Q, Mohamed A W, Zhou X B, Zhang B, Deng W. Enhanced success history adaptive DE for parameter optimization of photovoltaic models. Complexity, 2021, 2021, 6660115.

[71] Meng A B, Li Z, Yin H, Chen S Z, Guo Z Z. Accelerating particle swarm optimization using crisscross search. Information Sciences, 2016, 329, 52-72.

[72] Patwal R S, Narang N. Crisscross PSO algorithm for multi-objective generation scheduling of pumped storage hydrothermal system incorporating solar units. Energy Conversion and Management, 2018, 169, 238-254.

[73] Kaur M, Dhillon J S, Kothari D P. Crisscross differential evolution algorithm for constrained hydrothermal scheduling. Applied Soft Computing, 2020, 93, 106393.

[74] Tizhoosh H R. Opposition-based learning: A new scheme for machine intelligence. Proceedings - International Conference on Computational Intelligence for Modelling, Control and Automation, CIMCA 2005 and International Conference on Intelligent Agents, Web Technologies and Internet, Vienna, Austria, 2005, 695-701.

[75] Wang H, Wu Z J, Rahnamayan S, Liu Y, Ventresca M. Enhancing particle swarm optimization using generalized opposition-based learning. Information Sciences, 2011, 181, 4699-4714.

[76] Wang H, Rahnamayan S, Wu Z J. Parallel differential evolution with self-adapting control parameters and generalized opposition-based learning for solving high-dimensional optimization problems. Journal of Parallel and Distributed Computing, 2013, 73, 62-73.

[77] Wang J. Enhanced differential evolution with generalised opposition-based learning and orientation neighbourhood mining. International Journal of Computing Science and Mathematics, 2015, 6, 49-58.

[78] Wang B. A novel artificial bee colony algorithm based on modified search strategy and generalized opposition-based learning. Journal of Intelligent \& Fuzzy Systems, 2015, 28, 1023-1037.

[79] Zhou X, Wu Z, Deng C, Peng H. Enhancing artificial bee colony algorithm with generalised opposition-based learning. International Journal of Computing Science and Mathematics, 2015, 6, 297-309.

[80] Meng A B, Chen Y C, Yin H, Chen S Z. Crisscross optimization algorithm and its application. Knowledge-Based Systems, 2014, 67, 218-229.

[81] Meng A B, Hu H W, Yin H, Peng X G, Guo Z Z. Crisscross optimization algorithm for large-scale dynamic economic dispatch problem with valve-point effects. Energy, 2015, 93, 2175-2190.

[82] Meng A B, Mei P, Yin H, Peng X G, Guo Z Z. Crisscross optimization algorithm for solving combined heat and power economic dispatch problem. Energy Conversion and Management, 2015, 105, 1303-1317.

[83] Meng A B, Li J B, Yin H. An efficient crisscross optimization solution to large-scale non-convex economic load dispatch with multiple fuel types and valve-point effects. Energy, 2016, 113, 1147-1161.

[84] Zhao D, Liu L, Yu F H, Heidari A A, Wang M J, Oliva D, Muhammad K, Chen H L. Ant colony optimization with horizontal and vertical crossover search: Fundamental visions for multi-threshold image segmentation. Expert Systems with Applications, 2021, 167,114122.

[85] Liu Y, Chong G S, Heidari A A, Chen H L, Liang G X, Ye X J, Cai Z N, Wangg M J. Horizontal and vertical crossover of Harris hawk optimizer with Nelder-Mead simplex for parameter estimation of photovoltaic models. Energy Conversion and Management, 2020, 223, 113211.

[86] Demšar J. Statistical comparisons of classifiers over multiple data sets. The Journal of Machine Learning Research, 2006, 7, 1-30.

[87] Derrac J, Garcia S, Molina D, Herrera F. A practical tutorial on the use of nonparametric statistical tests as a methodology for comparing evolutionary and swarm intelligence algorithms. Swarm and Evolutionary Computation, 2011, 1, 3-18.

[88] Awad N H, Ali M Z, Suganthan P N, Liang J J, Qu B Y. Problem Definitions and Evaluation Criteria for the CEC 2017 Special Session and Competition on Single Objective Real-Parameter Numerical Optimization, technical report, 
Nanyang Technological University, Singapore, Jordan University of Science and Technology, Jordan, Zhengzhou University, China, 2016.

[89] Huang G B, Zhou H M, Ding X J, Zhang R. Extreme learning machine for regression and multiclass classification. IEEE Transactions on Systems, Man, and Cybernetics, Part B (Cybernetics), 2012, 42, 513-529.

[90] Zhu W, Ma C, Zhao X H, Wang M J, Heidari A A, Chen H L, Li C Y. Evaluation of sino foreign cooperative education project using orthogonal sine cosine optimized kernel extreme learning machine. IEEE Access, 2020, 8, 61107-61123.

[91] Chen H, Zhang Q, Luo J, Xu Y, Zhang X. An enhanced bacterial foraging optimization and its application for training kernel extreme learning machine. Applied Soft Computing, 2020, 86, 105884.

[92] Wang X C, Wang Z Y, Weng J, Wen C C, Chen H L, Wang X Q. A new effective machine learning framework for sepsis diagnosis. IEEE Access, 2018, 6, 48300-48310.

[93] Li Q, Chen H L, Huang H, Zhao X H, Cai Z G, Tong C F, Liu W B, Tian X. An enhanced grey wolf optimization based feature selection wrapped kernel extreme learning machine for medical diagnosis. Computational and Mathematical Methods in Medicine, 2017, 2017, 9512741.

[94] Chen H L, Wang G, Ma C, Cai Z N, Liu W B, Wang S J. An efficient hybrid kernel extreme learning machine approach for early diagnosis of Parkinson's disease. Neurocomputing, 2016, 184, 131-144.

[95] Luo J, Chen H, Zhang Q, Xu Y, Huang H, Zhao X. An improved grasshopper optimization algorithm with application to financial stress prediction. Applied Mathematical Modelling, 2018, 64, 654-668.

[96] Zhao D, Huang C Y, Wei Y, Yu F H, Wang M J, Chen H L. An effective computational model for bankruptcy prediction using kernel extreme learning machine approach. Computational Economics, 2017, 49, 325-341.

[97] Zhao X, Li D, Yang B, Liu S, Pan Z, Chen H. An efficient and effective automatic recognition system for online recognition of foreign fibers in cotton. IEEE Access, 2016, 4, 8465-8475.

[98] Huang G B, Zhu Q Y, Siew C K. Extreme learning machine: A new learning scheme of feedforward neural networks. IEEE International Joint Conference on Neural Networks, 2004, 982, 985-990.

[99] Sun Y, Xue B, Zhang M, Yen G G. Evolving deep convolutional neural networks for image classification. IEEE Transactions on Evolutionary Computation, 2020, 24,
394-407.

[100] Sun Y, Xue B, Zhang M, Yen G G, Lv J. Automatically designing $\mathrm{CNN}$ architectures using the genetic algorithm for image classification. IEEE Transactions on Cybernetics, 2020, 50, 3840-3854.

[101] Chang C C, Lin C J. LIBSVM: A library for support vector machines. ACM Transactions on Intelligent Systems and Technology, 2011, 2, https://doi.org/10.1145/1961189. 1961199.

[102] Xia J F, Chen H L, Li Q, Zhou M D, Chen L M, Cai Z N, Fang Y, Zhou H. Ultrasound-based differentiation of malignant and benign thyroid nodules: An extreme learning machine approach. Computer Methods and Programs in Biomedicine, 2017, 147, 37-49.

[103] Heidari A A, Abbaspour R A, Chen H L. Efficient boosted grey wolf optimizers for global search and kernel extreme learning machine training. Applied Soft Computing, 2019, 81, 105521.

[104] Wang S J, Chen H L, Yan W J, Chen Y H, Fu X L. Face recognition and micro-expression recognition based on discriminant tensor subspace analysis plus extreme learning machine. Neural Processing Letters, 2014, 39, 25-43.

[105] Wang S J, He Y, Li J, Fu X. MESNet: A convolutional neural network for spotting Multi-scale micro-expression Intervals in long videos. IEEE Transactions on Image Processing, 2021, 30, 3956-3969.

[106] Sun X, Liu Y, Wei D, Xu M, Chen H, Han J. Selection of interdependent genes via dynamic relevance analysis for cancer diagnosis. Journal of Biomedical Informatics, 2013, 46, 252-258.

[107] Chen H L, Wang G, Ma C, Cai Z N, Liu W B, Wang S J. An efficient hybrid kernel extreme learning machine approach for early diagnosis of Parkinson's disease. Neurocomputing, 2016, 184, 131-144.

[108] Liu D, Wang S S, Huang D Z, Deng G, Zeng F T, Chen H L. Medical image classification using spatial adjacent histogram based on adaptive local binary patterns. Computers in Biology and Medicine, 2016, 72, $185-200$.

[109] Zhao H L, Qiu X Q, Lu W L, Huang H, Jin X G. High-quality retinal vessel segmentation using generative adversarial network with a large receptive field. International Journal of Imaging Systems and Technology, 2020, 30, 828-842.

[110] Fei X Y, Wang J, Ying S H, Hu Z Y, Shi J. Projective parameter transfer based sparse multiple empirical kernel learning machine for diagnosis of brain disease. 
Neurocomputing, 2020, 413, 271-283.

[111] Li Y, Liu L, Guo Y, Liao X, Hu B, Yu T. Spatio-temporal-spectral hierarchical graph convolutional network with semi-supervised active learning for patient-specific seizure prediction. IEEE Transactions on Cybernetics, 2021, doi: https://doi.org/10.1109/TCYB.2021.3071860.

[112] Zhou Y W, Yang B, Hou H X, Zhang L N, Wang T, $\mathrm{Hu}$ M X. Continuous leakage-resilient identity-based encryption with tight security. Computer Journal, 2019, 62, $1092-1105$.

[113] Hu M X, Zhou Y, Li X J. Robust and accurate computation of geometric distance for Lipschitz continuous implicit curves. Visual Computer, 2017, 33, 937-947.

[114] Chen Z G, Lu M, Zhou Y M, Chen C Y. Information synergy entropy based multi-feature information fusion for the operating condition identification in aluminium electrolysis. Information Sciences, 2021, 548, 275-294.

[115] Zhao H L, Guo H Y, Jin X G, Shen J B, Mao X Y, Liu J R. Parallel and efficient approximate nearest patch matching for image editing applications. Neurocomputing, 2018, 305, 39-50.

[116] Li X J, Huang H, Zhao H L, Wang Y D, Hu M X. Learning a convolutional neural network for propagation-based stereo image segmentation. Visual Computer, 2020, 36, 39-52.

[117] Huang H, Li X J, Zhao H L, Nie G Z, Hu Z Y, Xiao L. Manifold-preserving image colorization with nonlocal estimation. Multimedia Tools and Applications, 2015, 74, 7555-7568.

[118] Wen F H, Yang X, Gong X, Lai K K. Multi-scale volatility feature analysis and prediction of gold price. International Journal of Information Technology \& Decision Making, 2017, 16, 205-223.

[119] Zhou P, Chen J Y, Fan M Y, Du L, Shen Y D, Li X J. Unsupervised feature selection for balanced clustering. Knowledge-Based Systems, 2020, 193, 105417.

[120] Li G L, Braysy O, Jiang L X, Wu Z D, Wang Y Z. Finding time series discord based on bit representation clustering. Knowledge-Based Systems, 2013, 54, 243-254.

[121] Guan R, Zhang H, Liang Y, Giunchiglia F, Huang L, Feng X. Deep feature-based text clustering and its explanation. IEEE Transactions on Knowledge and Data Engineering, 2020, https://doi.org/10.1109/TKDE.2020.3028943.

[122] Liu W H, Chang X J, Chen L, Phung D, Zhang X Q, Yang Y, Hauptmann A G. Pair-based uncertainty and diversity promoting early active learning for person re-identification.
Acm Transactions on Intelligent Systems and Technology, 2020, 11, 1-15.

[123] Yan C, Chang X, Luo M, Zheng Q, Zhang X, Li Z, Nie F. Self-weighted robust LDA for multiclass classification with edge classes. ACM Transactions on Intelligent Systems and Technology (TIST), 2020, 12, 1-19.

[124] Xue X, Chen Z, Wang S, Feng Z, Duan Y, Zhou Z. Value entropy: A systematic evaluation model of service ecosystem evolution. IEEE Transactions on Services Computing, 2020, doi: https://doi.org/10.1109/ TSC.2020.3016660.

[125] Feng C, Zhu Z, Cui Z, Ushakov V, Dreher J C, Luo W, Gu R, $\mathrm{Wu} X$, Krueger F. Prediction of trust propensity from intrinsic brain morphology and functional connectome. Human Brain Mapping, 2021, 42, 175-191.

[126] Wang H L, Wu X, Yao L. Identifying cortical brain directed connectivity networks from high-density EEG for emotion recognition. IEEE Transactions on Affective Computing, $2020,3006847$.

[127] Jin L, Wen Z J, Hu Z Y. Topology-preserving nonlinear shape registration on the shape manifold. Multimedia Tools and Applications, 2021, 80, 17377-17389.

[128] Yang C, Zhao H, Bruzzone L, Benediktsson J A, Liang Y, Liu B, Zeng X, Guan R, Li C, Ouyang Z. Lunar impact crater identification and age estimation with Chang'E data by deep and transfer learning. Nature Communication, 2020, 11, 6358.

[129] Cao X, Cao T, Gao F, Guan X. Risk-averse storage Planning for improving RES hosting capacity under uncertain siting choice. IEEE Transactions on Sustainable Energy, 2021, https://doi.org/10.1109/TSTE.2021.3075615.

[130] Fan M, Zhang X, Hu J, Gu N, Tao D. Adaptive data structure regularized multiclass discriminative feature selection. IEEE Transactions on Neural Networks and Learning Systems, 2021, https://doi.org/ 10.1109/TNNLS.2021.3071603.

[131] Zhang X Q, Fan M Y, Wang D, Zhou P, Tao D C. Top-k feature selection framework using robust $0-1$ integer programming. IEEE Transactions on Neural Networks and Learning Systems, 2020, 32, 3005-3019.

[132] Zhang X Q, Li W, Ye X Z, Maybank S. Robust hand tracking via novel multi-cue integration. Neurocomputing, 2015, 157, 296-305.

[133] Fan M Y, Zhang X Q, Chen S Y, Bao H J, Maybank S. Dimension estimation of image manifolds by minimal cover approximation. Neurocomputing, 2013, 105, 19-29.

[134] Wang T, Zhao L, Huang P C, Zhang X Q, Xu J W. Haze 
concentration adaptive network for image dehazing. Neurocomputing, 2021, 439, 75-85.

[135] Huang P C, Zhao L, Jiang R H, Wang T, Zhang X Q. Self-filtering image dehazing with self-supporting module. Neurocomputing, 2021, 432, 57-69.

[136] Zhang X Q, Wang T, Wang J X, Tang G Y, Zhao L. Pyramid channel-based feature attention network for image dehazing.
Computer Vision and Image Understanding, 2020, 197, 103003.

[137] Zhang X Q, Wang T, Luo W H, Huang P C. Multi-level fusion and attention-guided $\mathrm{CNN}$ for image dehazing. IEEE Transactions on Circuits and Systems for Video Technology, 2020, https://doi.org/10.1109/TCSVT.2020. 3046625 . 